\title{
Predictive Models of Clinical Improvement in Rituximab-Treated Myositis Patients Using Clinical Features, Autoantibodies, and Biomarkers
}

Jeannette M. Olazagasti' ${ }^{1}$, Cynthia S. Crowson ${ }^{1,2}$, Molly S. Hein' ${ }^{1}$, Consuelo Lopez de Padilla ${ }^{1}$, Rohit Aggarwal ${ }^{3,4}$, Chester V. Oddis ${ }^{3,4}$, Ann M. Reed ${ }^{*}$

\author{
${ }^{1}$ Division of Rheumatology, Mayo Clinic, Rochester, MN, USA \\ ${ }^{2}$ Division of Health Sciences Research, Mayo Clinic, Rochester, MN, USA \\ ${ }^{3}$ Division of Rheumatology, University of Pittsburgh, Pittsburgh, PA, USA \\ ${ }^{4}$ Division of Rheumatology, Department of Pediatrics, Duke University School of Medicine, Durham, NC, USA \\ Email: olazagastilourido.jeannette@mayo.edu, crowson@mayo.edu, hein.molly@mayo.edu, \\ lopezdepadilla.consuelo@mayo.edu, aggarwalr@upmc.edu, cvo5@pitt.edu, ${ }^{*}$ ann.reed@duke.edu
}

Received 29 May 2015; accepted 16 August 2015; published 19 August 2015

Copyright (C) 2015 by authors and Scientific Research Publishing Inc.

This work is licensed under the Creative Commons Attribution International License (CC BY). http://creativecommons.org/licenses/by/4.0/

(c) (i) Open Access

\section{Abstract}

Background: Response to rituximab so far is unpredictable in patients with refractory myositis. Predictive models of clinical improvement are developed using clinical, laboratory, and gene expression/cytokine/chemokine variables in rituximab-treated refractory myositis patients. Methods: We analyzed data for 200 myositis patients (76 with adult polymyositis (PM), 76 with adult dermatomyositis (DM), and 48 with juvenile (DM)) in the rituximab in myositis trial. Clinical improvement is defined as the change from baseline to 24 weeks in Physician Global Visual Analog Scale (VAS). We analyze the association of baseline variables with improvements: demographics, myositis subtype, clinical and laboratory parameters, autoantibody status, and interferon (IFN)regulated chemokines. Multivariable linear regression models are developed by using stepwise variable selection methods. Results: A "base" multivariable model to predict improvement with clinical and laboratory variablesonly is built with modest predictive ability (adjusted $R^{2}=0.21$ ). This model includes two significant factors at baseline: Physician Global VAS and Muscle Disease Activity VAS. A "final" multivariable model to predict improvement including non-standard laboratory measures is developed and demonstrated better predictive ability (adjusted $R^{2}=0.32$ ). This model includes Physician Global VAS, IFN chemokine score and IL-2 levels. The "final" model explained 11\% more variability than the "base" model. Conclusions: Changes in disease activity over time following treatment with rituximab in refractory myositis can be predicted. These mod-

\footnotetext{
"Corresponding author.
}

How to cite this paper: Olazagasti, J.M., et al. (2015) Predictive Models of Clinical Improvement in Rituximab-Treated Myositis Patients Using Clinical Features, Autoantibodies, and Biomarkers. Open Journal of Rheumatology and Autoimmune Diseases, 5, 68-80. http://dx.doi.org/10.4236/ojra.2015.53012 
els can be clinically useful to optimize treatment selection in myositis.

Keywords

Myositis, Rituximab, Cytokines, Chemokines

\section{Introduction}

The idiopathic inflammatory myopathies (IIMs) are a heterogeneous group of chronic acquired disorders characterized by proximal muscle weakness and muscle inflammation [1] [2]. In adults, the most common IIMs are dermatomyositis (DM) and polymyositis (PM) while in children, the most common IIM is juvenile DM [3]. Corticosteroids are the standard first-line therapy alone or in combination with immunosuppressive agents [4]. Unfortunately, many patients are refractory to corticosteroids and immunosuppressive agents, and therefore newer modes of therapy are currently being studied.

B cell depletion therapy with rituximab, which has been used for many years to treat B-cell lymphomas, has recently gained popularity in the treatment of autoimmune diseases. The prospective, randomized, double-blind, clinical trial (the Rituximab in Myositis, (RIM) trial) assesses the effectiveness of rituximab in refractory adult PM and adult and juvenile DM [5]. Although the RIM trial does not meet its primary or secondary end-points, $83 \%$ of study patients achieve the definition of improvement, and individual core set measures are improved in both rituximab-treated groups throughout the 44-week trial. Furthermore, the addition of rituximab provides a significant steroid-sparing effect between the start and conclusion of the trial [5]. However, there is a variation in the clinical response to rituximab among the patients that participate in the trial. Prediction of response to rituximab can prevent further exposing patients to side effects of rituximab. While rituximab is generally well tolerated and serious adverse reactions are uncommon, approximately 32\% of patients in the RIM trial and about $22 \%$ of patients in a trial of rituximab in vasculitis experience one or more adverse events [5] [6]. Identification of predictors of response to rituximab may also be helpful to develop recommendations for rituximab use, especially starting of rituximab as well as further continuation or early discontinuation.

Data regarding predictors of response to rituximab in refractory myositis is limited. Since particular clinical syndromes observed in myositis are closely linked to detection of myositis antibodies, Aggarwal et al. studies if autoantibodies can predict clinical improvement in rituximab-treated refractory myositis patients [7]. Their results indicate that anti-synthetase (mainly anti-Jo-1) and anti-Mi-2 autoantibodies are the strongest predictors of clinical improvement in rituximab-treated myositis patients [7]. Similarly, since inflammatory cytokines and chemokines have been shown to contribute to disease pathogenesis in myositis [8]-[11], Reed et al. explores whether a pre-treatment cytokine and chemokine profile help to identify IIM patients who respond favorably to rituximab therapy [12]. Their results indicate that the composite interferon (IFN) chemokine score before treatment can potentially predict response to rituximab in refractory myositis [12]. In light of these recent findings, we seek to determine clinical, laboratory, and gene expression/cytokine/chemokine predictors of clinical improvement in rituximab-treated refractory myositis patients, and explore whether the inclusion of gene expression, cytokine and chemokine data into the regression models offers a predictive advantage compared to models with clinical and laboratory data only.

\section{Methods}

\subsection{Patientsand Study Design}

This study enrolled 200 subjects with refractory adult $(n=76)$ and juvenile DM $(n=48)$ and adult PM (n = 76). All subjects were part of a previously reported multicenter clinical trial, RIM trial [5]. The RIM trial used a randomized, double-blind, placebo-phase design of intravenous rituximab in which refractory subjects were randomized to either an "early-start arm” (rituximab at weeks 0/1, placebo at weeks 8/9) or "late-start arm” (placebo at weeks 0/1, rituximab at weeks 8/9); therefore all subjects received rituximab. Demographic, clinical features, and the values for the core set measures at baseline were similar between the early and late rituximabtreated groups. There was no statistical difference in the time to achieving the definition of improvement in the 
adult PM, adult DM, and juvenile DM subsets. Baseline in our analyses was defined as the time of rituximab treatment initiation (i.e., week 0 for the early arm and week 8 for the late arm).

\subsection{Clinical Assessment}

Clinical assessment and disease activity measures were evaluated using a core set of measures (CSMs) described by the International Myositis Assessment and Clinical Studies Group (IMACS). These included Physician Global Visual Analogue Scale (VAS) and Extramuscular Global VAS. Extramuscular Global VAS uses a combined score based on the investigator's composite assessment of the Constitutional, Cutaneous, Skeletal, Gastrointestinal Disease, Pulmonary, Cardiac, and Muscle Disease Activity VAS of the Myositis Disease Activity Assessment Tool (MDAAT). All study participants had their disease activity assessed at the time of entry to the trial and at all follow up visits.

\subsection{Clinical Improvement}

Clinical improvement was based on absolute change from baseline to 24 weeks in three disease activity measures: Physician Global VAS, Muscle Disease Activity VAS, and Extramuscular Global VAS. We included in our analysis the Physician Global VAS and Muscle Disease Activity VAS specifically since Reed et al. [13] previously reported that these measures had the most correlation with the IFN chemokine score.

\subsection{Autoantibody Assessment}

Myositis specific antibodies were performed at the rheumatology research laboratory at the University of Pittsburgh using immunoprecipitation (IP) techniques as previously described [5]. Autoantibodies were classified into 8 groups: (1) anti-aminoacyl-transfer RNA synthetases (anti-Jo-1, anti-PL-7, anti-PL-12, anti-KS, anti-OJ, and anti-EJ); (2) anti-transcription intermediary factor $1 \gamma$ (anti-TIF-1 $\gamma$ ); (3) anti-SRP; (4) anti-MJ; (5) anti-Mi-2; (6) other known autoantibodies seen in myositis (anti-PM-Scl, anti-U1 RNP, anti-SSA/SSB, anti-Ku, anti-SAE, anti-U1/U2, and anticentromere antibody); (7) undefined autoantibodies (i.e., those that could not be definitively identified by IP); and (8) patients with no detectable autoantibodies.

\subsection{Measurement of Gene Expression}

Samples of whole blood were obtained from subjects and drawn into PAXgene tubes (Qiagen/Becton Dickinson, Franklin Lakes, NJ). Total RNA was isolated from the blood with on-column DNase treatment, according to the manufacturer's protocol. The RNA yield and integrity were assessed using an Agilent Lab-on-a-Chip Bioanalyzer (Agilent Technologies, Palo Alto, CA). The type I IFN gene expression signature was defined in the whole blood by determining the expression levels of 3 IFN-regulated genes (IFIT1, G1P2, and IRF7), as measured by TaqMan quantitative real-time reverse transcription-polymerase chain reaction using the ABI Prism 7900HT Sequence Detection System (Applied Biosystems, Foster City, CA). Relative quantification of the gene expression levels was performed by comparison of the values against a calibrator sample (PAXgene whole blood RNA from a healthy control subject), in accordance with the manufacturer's guidelines, and the results were normalized to the values for GAPDH. For each gene, the 95th percentile of expression levels was calculated. Expression values equal to or greater than the 95th percentile were replaced with the 95th percentile value and then normalized, so that the maximum value for each gene was 1.0 [14]. Finally, the normalized expression values for the 3 genes were then summed for each patient, and the sums were adjusted to a 100-point scale, to yield the summary type I IFN gene score.

\subsection{Measurement of Serum Cytokines and Chemokines}

Serum was isolated from blood drawn into Serum-Separator Tubes (BD Vacutainer ${ }^{\circledR}$ Blood Collection Tube; Becton, Dickinson, USA at baseline and 8 and 24 weeks after the first dose of rituximab). A protease inhibitor (aprotinin; $1 \mu \mathrm{g} / \mathrm{ml}$ ) was added to each sample, and aliquots were immediately frozen at $-80^{\circ} \mathrm{C}$. Multiplexed sandwich immunoassays (Meso Scale Discovery, Rockville, MD) were used to quantitate the serum levels of IFN regulated chemokines [monokine induced by gamma interferon (MIG/CXCL9), macrophage inflammatory 
protein-1 $\alpha$ and $\beta$ (MIP-1 $\alpha$ /CCL3 and MIP-1//CCL4), monocyte chemotactic protein-1 and 2 (MCP-1 and MCP-2), interferon gamma-induced protein 10 (IP-10/CXCL10), interferon-inducible T-cell alpha chemoattractant (I-TAC/CXCL11), TNF receptor type I (TNFR1), and Interferon $\alpha$ and $\gamma$ (IFN $\alpha$, IFN $\gamma$ ), and the serum levels of tumor necrosis factor $\alpha$ (TNF- $\alpha$ ), interleukin-2 (IL-2), IL-1 $\beta$, IL-4, IL-5 IL-6, IL-8, IL-10, IL-12, IL-13, and IL-17. This panel of cytokines/chemokines was selected a priori [9] [11] [15]-[18]. Samples were run in duplicate and calibrated recombinant proteins were used to generate standard curves.

A composite IFN chemokine score was generated based on serum levels of 3 IFN-regulated chemokines (IP-10, I-TAC and MCP-1), which exhibited the strongest correlations with disease activity in adult and juvenile DM in our previous report [16]. The IFN chemokine scores were determined by first calculating the 95th percentile of serum concentration levels for each chemokine and then setting concentration levels $\geq 95$ th percentile to the 95th percentile value to reduce extreme outliers as previously described [10]. For each chemokine, the data were re-scaled to a maximum value of 1.0. For each subject, the re-scaled values were summed up, and the sum was adjusted to a 100 -point scale.

\subsection{Baseline Predictor Variables}

Baseline clinical, laboratory and gene expression/cytokine/chemokine variables of interest were selected a priori based on clinical experience and previous studies. The variables considered as potential predictors of clinical improvement after rituximab therapy are listed in Table 1.

\subsection{Statistical Methods}

Baseline potential predictor variables were univariately assessed for association with clinical improvement using non-parametric Spearman correlation methods for continuous variables and Mann-Whitney tests for categorical variables. Univariate factors with a $\mathrm{P}$ value of $<0.15$ were combined using a multivariable linear regression model. Within the model, individual factors were tested at the 0.05 significance level. The predictive ability of the resulting models was expressed as a percentage of variance explained by the model as measured by the adjusted $\mathrm{R}^{2}$, where the adjustment accounts for the number of variables included in the model. Models with adjusted $\mathrm{R}^{2}>0.30$ were considered to have good predictive ability. In order to investigate whether inclusion of gene expression/cytokine/chemokine data into the models provided a predictive advantage, we performed our multivariable analyses both without gene expression/cytokine/chemokine data ("base models"), and with gene expression/cytokine/chemokine data ("final" models). To quantify the improvement in comparison with the "base" models, the difference in adjusted $\mathrm{R}^{2}$ between the "final" models and the "base" models were used as this is the amount of additional variation in the outcome measure that is explained by the "final" model compared to the "base" model. However, stepwise variable selection methods are known to be biased and often result in over fitting, so Least Absolute Shrinkage and Selection Operator (LASSO)-penalized regression methods were also

Table 1. Baseline predictor variables analyzed for univariate analysis.

\begin{tabular}{|c|c|}
\hline Group & Variables \\
\hline Demographic features & Age at diagnosis, sex, race, and time to diagnosis \\
\hline Myositis subgroup & Polymyositis, dermatomyositis, or juvenile dermatomyositis \\
\hline Medication & Prednisone use, DMARD use, and number of total failed immunosuppressive agents at trial entry \\
\hline Clinical parameters & $\begin{array}{c}\text { Physician's Global Assessment, Muscle Disease Activity, Extramuscular Global Assessment, } \\
\text { Constitutional Disease Activity, Cutaneous Disease Activity, Skeletal Disease Activity, } \\
\text { Pulmonary Disease Activity, Patient’s/Parent’s Global Assessment, Manual Muscle } \\
\text { Testing (MMT8), HAQ/C-HAQ Disability Index, and Overall Damage Score }\end{array}$ \\
\hline Laboratory parameters & Lymphocyte count, ALT levels, AST levels, aldolase levels, CPK levels, total IgM and IgG levels \\
\hline Autoantibody groups & $\begin{array}{l}\text { Antisynthetase, anti-TIF- } 1 \gamma \text {,anti-SRP, anti-MJ, anti-Mi-2, other autoantibodies, } \\
\text { no autoantibodies, or unspecified autoantibodies }\end{array}$ \\
\hline $\begin{array}{l}\text { Gene Expression/ } \\
\text { Cytokines/Chemokines }\end{array}$ & $\begin{array}{l}\text { IFN gene score, IFN chemokine score, IFN- } \gamma \text {, IFN- } \alpha \text {, IL-10, IL-12, IL-13, IL-1 } \beta \text {, IL-2, IL-4, IL-5, } \\
\text { IL-8, IL-6, IL-17, IP-10, TNF- } \alpha \text {, MIP-1 } \alpha \text {, MIP-1 } \beta \text {, ITAC, MCP-1, MCP-2, MIG, and TNFR1 }\end{array}$ \\
\hline
\end{tabular}


used for variable selection [19]. Internal validation using 10-fold cross validation is built into the LASSO software to reduce over-fitting and more accurately estimate how the model predictions will perform on new data.

Analyses were performed using JMP 11.2.1 (JMP Statistical Discovery, North Carolina, USA) and R 3.0.2 (R Foundation for Statistical Computing, Vienna, Austria) statistical software.

\section{Results}

\subsection{Patient Characteristics}

Detailed data on subjects' demographics, baseline disease characteristics, safety and clinical outcomes of the RIM trial were previously reported [5]. Briefly, most subjects were Caucasian (70\%) and female (73\%), with longstanding disease duration (mean $(\mathrm{SD})=5.4$ [6.5] years) and highly active disease as evidenced by the Physician Global (mean (SD) = 49 [19] $\mathrm{mm}$ ) and Muscle Disease Activity VAS scores (mean (SD) = 46 [22] mm). Subjects had failed a mean of 3.1 immunosuppressive agents in addition to glucocorticoids [5]. The average prednisone dosage at study entry was $21 \mathrm{mg} /$ day.

The following autoantibody profiles were detected: antisynthetase $n=28$, TIF- $1 \gamma n=19$, SRP $n=21$, Mi-2 $n$ $=25$, MJ $n=18$, other autoantibodies $n=24$, unidentified autoantibodies $n=9$ and no autoantibodies $n=33$. Cytokine and chemokine analysis were available only for 177 of 200 subjects from the RIM trial.

\subsection{Univariate Analyses of Baseline Patient Characteristics That Associated with Change in Disease Activity Measures}

\subsubsection{Physician Global VAS}

The results of the univariate analyses of 63potential predictor variables are summarized in Table 2. Nine clinical and laboratory variables were identified as primary candidates for inclusion in the Physician Global VAS "base" multivariable model $(P<0.15)$. Of these, the following 8baseline variables had a significant $(P<0.05)$ univariate association with clinical improvement in Physician Global VAS: Physician Global VAS, Muscle Disease Activity VAS, Extramuscular Global VAS, Constitutional Disease Activity VAS, Cutaneous Disease Activity VAS, Patient/Parent Global VAS, lymphocyte count, and AST levels.

Ninegene expression/cytokine/chemokine variables were identified as primary candidates for inclusion in the Physician Global VAS "final” multivariable model $(P<0.15)$. The following 7 variables had a significant $(P<$ $0.05)$ univariate association with clinical improvement in Physician Global VAS: IFN gene score, IFN chemokine score, IP-10, TNF- $\alpha$, ITAC, MCP-1, and MCP-2.

\subsubsection{Muscle Disease Activity VAS}

Ten clinical and laboratory variables were identified as primary candidates for inclusion in the Muscle Disease Activity VAS "base" multivariable model $(P<0.15)$. Of these, the following 6 variables had a significant $(P<$ 0.05 ) univariate association with clinical improvement in Muscle Disease Activity VAS: Physician Global VAS, Muscle Disease Activity VAS, Extramuscular Global VAS, lymphocyte count, AST levels, and presence of antiTIF- $1 \gamma$.

Fifteen gene expression/cytokine/chemokine variables were identified as primary candidates for inclusion in the Muscle Disease Activity VAS "final" multivariable model $(P<0.15)$. The following 6 variables had a significant $(P<0.05)$ univariate association with clinical improvement in Muscle Disease Activity VAS: IFN gene score, IFN chemokine score, IP-10, TNF- $\alpha$, ITAC, and MCP-1.

\subsubsection{Extramuscular Global VAS}

Fifteen clinical and laboratory variables were identified as primary candidates for inclusion in the Extramuscular Global VAS "base” multivariable model $(P<0.15)$. The following 8 variables had a significant $(P<0.05)$ univariate association with clinical improvement in Extramuscular Global VAS: Physician Global VAS, Muscle Disease Activity VAS, Extramuscular Global VAS, Constitutional Disease Activity VAS, Cutaneous Disease Activity VAS, Health Assessment Questionnaire (HAQ) Disability Index for adults and Children's Health Assessment Questionnaire (C-HAQ) Disability Index for pediatric patients, AST levels, and total IgM levels.

Ninegene expression/cytokine/chemokine variables were identified as primary candidates for inclusion in the Extramuscular Global VAS “final” multivariable model $(P<0.15)$. The following 7 variables had a significant 
Table 2. Baseline univariate variables and their association with the level of response for Physician Global VAS, Muscle Disease Activity VAS and Extramuscular Global VAS.

\begin{tabular}{|c|c|c|c|c|c|c|}
\hline \multirow[t]{2}{*}{ Variables } & \multicolumn{2}{|c|}{$\begin{array}{l}\text { Absolute change in Physician } \\
\text { Global VAS } 24 \text { weeks after } \\
\text { treatment } \\
(n=177)\end{array}$} & \multicolumn{2}{|c|}{$\begin{array}{l}\text { Absolute change in Muscle } \\
\text { Disease Activity VAS } 24 \\
\text { weeks after treatment } \\
(n=177)\end{array}$} & \multicolumn{2}{|c|}{$\begin{array}{l}\text { Absolute change in } \\
\text { Extramuscular Global } \\
\text { VAS } 24 \text { weeks after } \\
\text { treatment } \\
(n=177)\end{array}$} \\
\hline & $\begin{array}{l}\text { Correlation } \\
\text { Coefficient }\end{array}$ & P-value & $\begin{array}{l}\text { Correlation } \\
\text { Coefficient }\end{array}$ & P-value & $\begin{array}{c}\text { Correlation } \\
\text { Coefficient }\end{array}$ & P-value \\
\hline \multicolumn{7}{|l|}{ Demographic features } \\
\hline Age at diagnosis, years & -0.03 & 0.71 & -0.07 & 0.35 & 0.01 & 0.94 \\
\hline Time to diagnosis, years & 0.09 & 0.21 & 0.04 & 0.54 & -0.01 & 0.85 \\
\hline Male sex & 1.23 & 0.27 & 0.04 & 0.84 & 0.33 & 0.57 \\
\hline Race, Caucasian & 0.56 & 0.45 & 2.40 & 0.12 & 0.29 & 0.59 \\
\hline Disease subset, juvenile DM & 0.19 & 0.67 & 0.06 & 0.81 & $<0.01$ & 0.99 \\
\hline Disease subset, adult DM & 0.35 & 0.55 & 0.89 & 0.35 & 1.92 & 0.17 \\
\hline \multicolumn{7}{|l|}{ Medication } \\
\hline Prednisone use & 0.29 & 0.59 & 0.71 & 0.40 & 1.73 & 0.19 \\
\hline DMARD use & 1.22 & 0.27 & 0.48 & 0.49 & 1.03 & 0.31 \\
\hline Number of failed immunosuppressive agents & 0.02 & 0.82 & 0.02 & 0.79 & -0.11 & 0.15 \\
\hline \multicolumn{7}{|l|}{ Clinical parameters } \\
\hline Physician’s Global Assessment VAS & -0.40 & $<0.01$ & -0.37 & $<0.01$ & -0.21 & $<0.01$ \\
\hline Muscle Disease Activity VAS & -0.28 & $<0.01$ & -0.42 & $<0.01$ & -0.16 & 0.03 \\
\hline Extramuscular Global Assessment VAS & -0.24 & $<0.01$ & -0.21 & $<0.01$ & -0.57 & $<0.01$ \\
\hline Constitutional Disease Activity VAS & -0.16 & 0.03 & -0.14 & 0.06 & -0.34 & $<0.01$ \\
\hline Cutaneous Disease Activity VAS & -0.19 & 0.01 & -0.13 & 0.08 & -0.29 & $<0.01$ \\
\hline Skeletal Disease Activity VAS & -0.06 & 0.41 & -0.05 & 0.51 & -0.13 & 0.08 \\
\hline Pulmonary Disease Activity VAS & 0.01 & 0.93 & 0.07 & 0.36 & -0.12 & 0.11 \\
\hline Patient’s/parent's Global Assessment VAS & -0.16 & 0.03 & -0.09 & 0.20 & -0.12 & 0.09 \\
\hline Manual Muscle Testing (MMT8) & 0.08 & 0.29 & 0.10 & 0.19 & 0.11 & 0.15 \\
\hline HAQ/C-HAQ Disability Index & -0.05 & 0.49 & -0.09 & 0.20 & -0.16 & 0.03 \\
\hline Overall Damage Score & -0.02 & 0.77 & -0.02 & 0.74 & -0.06 & 0.42 \\
\hline \multicolumn{7}{|l|}{ Laboratory parameters } \\
\hline Lymphocyte count & 0.21 & $<0.01$ & 0.17 & 0.02 & 0.11 & 0.13 \\
\hline ALT & -0.08 & 0.29 & -0.05 & 0.46 & -0.01 & 0.85 \\
\hline AST & -0.21 & $<0.01$ & -0.15 & 0.04 & -0.16 & 0.03 \\
\hline Aldolase & -0.10 & 0.20 & -0.09 & 0.24 & -0.03 & 0.72 \\
\hline CPK & -0.07 & 0.33 & -0.06 & 0.46 & $<0.01$ & 0.97 \\
\hline Total IgG & -0.11 & 0.13 & -0.06 & 0.39 & -0.11 & 0.12 \\
\hline Total IgM & -0.10 & 0.18 & -0.11 & 0.14 & -0.17 & 0.02 \\
\hline Autoantibody groups & & & & & & \\
\hline
\end{tabular}




\section{Continued}

\begin{tabular}{|c|c|c|c|c|c|c|}
\hline Antisynthetase & 1.63 & 0.20 & 0.09 & 0.76 & 0.72 & 0.40 \\
\hline Anti-TIF- $1 \gamma$ & 1.76 & 0.18 & 4.78 & 0.03 & 0.06 & 0.81 \\
\hline Anti-SRP & 1.95 & 0.16 & 1.01 & 0.31 & 0.58 & 0.45 \\
\hline Anti-MJ & 1.76 & 0.19 & 0.65 & 0.42 & 0.85 & 0.36 \\
\hline Anti-MI-2 & 1.95 & 0.16 & 0.38 & 0.54 & 2.86 & 0.09 \\
\hline Other autoantibodies & 0.08 & 0.78 & 0.02 & 0.89 & 0.05 & 0.83 \\
\hline Unspecified autoantibodies & 0.95 & 0.33 & 1.73 & 0.19 & 2.98 & 0.08 \\
\hline \multicolumn{7}{|c|}{ Gene expression/Cytokines/Chemokines } \\
\hline IFN gene score & -0.21 & 0.01 & -0.17 & 0.04 & -0.18 & 0.03 \\
\hline IFN chemokine score & -0.30 & $<0.01$ & -0.19 & 0.01 & -0.22 & $<0.01$ \\
\hline IFN- $\gamma$ & -0.04 & 0.57 & -0.15 & 0.05 & 0.13 & 0.09 \\
\hline IFN- $\alpha$ & -0.06 & 0.47 & -0.06 & 0.52 & 0.05 & 0.60 \\
\hline IL-10 & -0.10 & 0.19 & -0.12 & 0.13 & 0.01 & 0.88 \\
\hline IL-12 & 0 & 0.99 & -0.06 & 0.43 & 0.05 & 0.54 \\
\hline IL-13 & -0.08 & 0.32 & -0.09 & 0.26 & -0.06 & 0.40 \\
\hline IL- $1 \beta$ & -0.03 & 0.70 & -0.09 & 0.22 & -0.03 & 0.66 \\
\hline IL-2 & -0.12 & 0.11 & -0.14 & 0.07 & 0.05 & 0.48 \\
\hline IL-4 & -0.05 & 0.53 & -0.09 & 0.24 & -0.04 & 0.64 \\
\hline IL-5 & -0.01 & 0.93 & -0.06 & 0.45 & 0.02 & 0.80 \\
\hline IL-8 & -0.03 & 0.67 & -0.02 & 0.81 & 0.04 & 0.57 \\
\hline IL-6 & -0.09 & 0.27 & -0.12 & 0.13 & -0.06 & 0.44 \\
\hline IL-17 & -0.03 & 0.67 & -0.04 & 0.57 & 0.02 & 0.76 \\
\hline IP-10 & -0.27 & $<0.01$ & -0.18 & 0.02 & -0.22 & $<0.01$ \\
\hline TNF- $\alpha$ & -0.17 & 0.02 & -0.18 & 0.02 & -0.05 & 0.55 \\
\hline MIP- $1 \alpha$ & -0.05 & 0.56 & -0.15 & 0.06 & -0.08 & 0.30 \\
\hline MIP- $1 \beta$ & -0.10 & 0.21 & -0.10 & 0.18 & -0.16 & 0.04 \\
\hline ITAC & -0.26 & $<0.01$ & -0.18 & 0.02 & -0.18 & 0.02 \\
\hline MCP-1 & -0.30 & $<0.01$ & -0.16 & 0.04 & -0.23 & $<0.01$ \\
\hline МСР-2 & -0.25 & $<0.01$ & -0.13 & 0.10 & -0.23 & $<0.01$ \\
\hline MIG & -0.14 & 0.06 & -0.08 & 0.33 & -0.06 & 0.41 \\
\hline TNFR1 & -0.13 & 0.16 & -0.16 & 0.08 & -0.05 & 0.61 \\
\hline
\end{tabular}

$(P<0.05)$ univariate association with clinical improvement in terms of Extramuscular Global VAS: IFN gene score, IFN chemokine score, IP-10, MIP-1 $\beta$, ITAC, MCP-1, and MCP-2.

\subsection{Multivariable Analysis of Baseline Patient Characteristics That Predicted Change in Disease Activity Measures}

3.3.1. Physician Global VAS “Base” Multivariable Model

A "base" multivariable model to predict level of response for Physician Global VAS with clinical and laboratory variables only (without gene expression/cytokine/chemokine data) was built with modest predictive ability (adjusted $\mathrm{R}^{2}=0.21$ ). This model for change in Physician Global VAS from baseline to week 24 included the following 2 significant factors at baseline: Physician Global VAS and Muscle Disease Activity VAS. The coeffi- 
cients and P-values for these 2 factors are summarized in Table 3.

\subsubsection{Physician Global VAS "Final” Multivariable Model}

A "final” multivariable model to predict level of response for Physician Global VAS including gene expression/ cytokine/chemokine data along with clinical and laboratory variables was built with good predictive ability (adjusted $\mathrm{R}^{2}=0.32$ ). The model for change in Physician Global VAS from baseline to week 24 included the following 3 significant factors at baseline: Physician Global VAS, IFN chemokine score, and IL-2 levels. The coefficients and P-values for these 3 factors are summarized in Table 4 and information on how to compute the scores can be found in Appendix. In comparison with the Physician Global "base" model, the gain in adjusted $\mathrm{R}^{2}$ of the Physician Global VAS "final" model was 0.11 . Therefore, the Physician Global VAS "final" model explained 11\% more of the variability in the change in Physician Global VAS than the "base" model. However, the penalized regression methods resulted in a multivariable model containing only Physician Global VAS and IFN chemokine score with a cross-validated $\mathrm{R}^{2}$ value of 0.13 (Table 5).

Table 3. "Base” multivariable models for predicting improvement (without gene expression/cytkokines/chemokine data).

\begin{tabular}{|c|c|c|c|c|c|c|}
\hline \multirow[t]{2}{*}{ Predictors } & \multicolumn{2}{|c|}{$\begin{array}{c}\text { Absolute change in Physician } \\
\text { Global VAS } 24 \text { weeks after } \\
\text { treatment }(n=177)\end{array}$} & \multicolumn{2}{|c|}{$\begin{array}{l}\text { Absolute change in Muscle } \\
\text { Disease Activity VAS } 24 \text { weeks } \\
\text { after treatment ( } n=177)\end{array}$} & \multicolumn{2}{|c|}{$\begin{array}{l}\text { Absolute change in Extra-muscular } \\
\text { Global VAS } 24 \text { weeks after treatment } \\
\qquad(n=177)\end{array}$} \\
\hline & Coefficient & P-value & Coefficient & P-value & Coefficient & P-value \\
\hline Intercept & -1.11 & - & 1.09 & - & -3.14 & - \\
\hline Race (Caucasian) & - & - & -4.67 & $<0.01$ & - & - \\
\hline Physician Global VAS & -0.67 & $<0.01$ & - & - & - & - \\
\hline Muscle Disease Activity VAS & 0.29 & $<0.01$ & -0.43 & $<0.01$ & - & - \\
\hline Extramuscular Global VAS & - & - & - & - & -0.52 & $<0.01$ \\
\hline Pulmonary Disease Activity VAS & - & - & - & - & 0.22 & $<0.01$ \\
\hline Lymphocyte count & - & - & - & - & 0.21 & 0.02 \\
\hline Adjusted $\mathrm{R}^{2}$ & 0.21 & - & 0.23 & - & 0.34 & - \\
\hline
\end{tabular}

Table 4. "Final” multivariable models for predicting improvement (with gene expression/cytokine/chemokine data).

\begin{tabular}{|c|c|c|c|}
\hline Predictors & $\begin{array}{c}\text { Absolute change in Physician } \\
\text { Global VAS } 24 \text { weeks after } \\
\text { treatment }(n=177)\end{array}$ & $\begin{array}{l}\text { Absolute change in Muscle } \\
\text { Disease Activity VAS } 24 \\
\text { weeks after treatment } \\
(n=177)\end{array}$ & $\begin{array}{l}\text { Absolute change in } \\
\text { Extra-muscular Global VAS } \\
24 \text { weeks after treatment } \\
(n=177)\end{array}$ \\
\hline & Coefficient & Coefficient & Coefficient \\
\hline Intercept & 1.36 & 6.06 & -4.32 \\
\hline Race (Caucasian) & - & -5.03 & - \\
\hline Physician Global VAS & -0.34 & - & - \\
\hline Muscle Disease Activity VAS & - & -0.40 & - \\
\hline Extramuscular Global VAS & - & - & -0.47 \\
\hline Pulmonary Disease Activity VAS & - & - & 0.20 \\
\hline Presence of anti-Mi-2 & - & - & -5.63 \\
\hline IFN chemokine score & -0.26 & -0.19 & - \\
\hline IL-2, pg/mL & 1.10 & 0.91 & - \\
\hline $\mathrm{MIP}-1 \beta, \mathrm{pg} / \mathrm{mL}$ & - & - & 0.004 \\
\hline Adjusted $\mathrm{R}^{2}$ & 0.32 & 0.33 & 0.42 \\
\hline
\end{tabular}


Table 5. Multivariable models for predicting improvement (with gene expression/cytokine/chemokine data) using penalized regression methods.

\begin{tabular}{cccc}
\hline Predictors & $\begin{array}{c}\text { Absolute change in Physician } \\
\text { Global VAS 24 weeks after } \\
\text { treatment (n= 177) }\end{array}$ & $\begin{array}{c}\text { Absolute change in Muscle } \\
\text { Disease Activity VAS 24 weeks } \\
\text { after treatment (n = 177) }\end{array}$ & $\begin{array}{c}\text { Extra-muscular Global VAS 24 } \\
\text { weeks after treatment } \\
\text { (n = 177) }\end{array}$ \\
Coefficient
\end{tabular}

\subsubsection{Muscle Disease Activity VAS "Base" Multivariable Model}

A "base" multivariable model to predict level of response for Muscle Disease Activity VAS without gene expression/cytokine/chemokine data was built with modest predictive ability (adjusted $\mathrm{R}^{2}=0.23$ ). The model for change in Muscle Disease Activity VAS from baseline to week 24 included the following 2 significant factors at baseline: Race (Caucasian) and Muscle Disease Activity VAS. The coefficients and P-values for these 2 factors are summarized in Table 3.

\subsubsection{Muscle Disease Activity VAS “Final” Multivariable Model}

A "final" multivariable model to predict level of response for Muscle Disease Activity VAS with gene expression/cytokine/chemokine data along with clinical and laboratory variables was built with good predictive ability (adjusted $\mathrm{R}^{2}=0.33$ ). The model for change in Muscle Disease Activity VAS from baseline to week 24 included the following 4 significant factors at baseline: Race (Caucasian), Muscle Disease Activity VAS, IFN chemokine score, and IL-2. The coefficients and P-values for these 4 factors are summarized in Table 4. In comparison with the Muscle Disease Activity VAS "base" model, the gain in adjusted $\mathrm{R}^{2}$ of the Muscle Disease Activity VAS "final" model was 0.10 . Therefore, the Muscle Disease Activity VAS "final" model explained $10 \%$ more variability than the "base" model. However, the penalized regression methods resulted in a multivariable model containing only Muscle Disease Activity VAS with a cross-validated $\mathrm{R}^{2}$ value of 0.05 (Table 5).

\subsubsection{Extramuscular Global VAS “Base" Multivariable Model}

A "base" multivariable model to predict level of response for Extramuscular Global VAS without gene expression/cytokine/chemokine data was built with good predictive ability (adjusted $\mathrm{R}^{2}=0.34$ ). The model for change in Extramuscular Global VAS from baseline to week 24 included 3 significant factors at baseline: lymphocyte count, Extramuscular Global VAS, and Pulmonary Disease Activity VAS. The coefficients and P-values for these 3 factors are summarized in Table 3.

\subsubsection{Extramuscular Global VAS "Final” Multivariable Model}

A "final" multivariable model to predict level of response for Extramuscular Global VAS with gene expression/ cytokine/chemokine data along with clinical and laboratory variables was built with good predictive ability (adjusted $\mathrm{R}^{2}=0.42$ ). The model for change in Extramuscular Global VAS from baseline to week 24 included the following 4significant factors at baseline: Extramuscular Global VAS, Pulmonary VAS, presence of anti-Mi-2, 
and MIP-1 $\beta$ levels. The coefficients and P-values for these 4 factors are summarized in Table 4. In comparison with the Extramuscular Global "base" model, the gain in adjusted $\mathrm{R}^{2}$ of the Extramuscular Global VAS "final" model was 0.08 . Therefore, the Extramuscular Global VAS "final" model explained 8\% more variability than the "base" model. However, the penalized regression methods resulted in a multivariable model containing only Extramuscular Global VAS with a cross-validated $\mathrm{R}^{2}$ value of 0.14 (Table 5).

\section{Discussion}

The recent RIM trial demonstrates that rituximab is an effective therapy for patients with treatment-resistant myositis [5]. However, rituximab does not benefit all patients in the RIM trial. To support an "individualized-medicine" approach to the treatment of myositis, the identification of clinical features and biomarkers that predict a beneficial response to rituximab is necessary. Herein, we seek to identify clinical, laboratory, and gene expression/cytokine/chemokine predictors of clinical improvement in a cohort of rituximab-treated refractory myositis. Interestingly, the "final" models that include gene expression/cytokine/chemokinedata are superior in terms of predictive ability when compared to the "base" models with only clinical and laboratory data. Our "final” models demonstrate that Physician Global VAS, IFN chemokine score, and IL-2 levels at baseline help predict to some extent Physician Global VAS at 24 weeks. Similarly, they demonstrate that race (Caucasian), Muscle Disease Activity VAS, IFN chemokine score and IL-2 levels at baseline help predict somewhat Muscle Disease Activity VAS at 24 weeks; and Extramuscular Global VAS, Pulmonary VAS, presence of anti-Mi-2, and MIP-1 $\beta$ levels at baseline help predict to a certain degree Extramuscular Global VAS at 24 weeks.

Cytokines and chemokines associated with innate and adaptive immune-pathways have emerged as important factors in the pathogenesis of inflammatory myopathies. Previous studies have reported up-regulation of numerous Type I IFN genes, cytokines, and chemokines in both peripheral blood and muscle tissue of PM and DM patients with active disease [13]. Nevertheless, in order to reduce comparisons of individual measurements as well as simplify comparisons across studies, composite scores using subsets of Type I IFN up-regulate genes, cytokines and chemokines have been developed. For example, the whole blood type I IFN gene expression signature is defined by expression levels of 3 IFN-regulated genes: IFIT1, G1P2, and IRF7 [10] [13]. In a manner similar to the calculation of the Type I IFN gene score, a summary chemokine score based on serum levels of IP-10, ITAC and MCP-1 has also been defined [13]. Interestingly, the composite Type I IFN gene and chemokine scores have been shown to be sensitive to change in myositis disease activity (especially the Physician Global VAS and Muscle Disease Activity VAS scores) [13]. Therefore, these composite scores have the potential to serve as sensitive and responsive longitudinal biomarkers of change in myositis disease activity.

Evidence suggests that an altered expression of cytokines and chemokines in both peripheral blood and muscle tissue of myositis patients may be particularly relevant in refractory disease. For example, expression of IL18 at in muscle from patients with myositis has been associated with treatment-resistant disease [20]. Similarly, expression of Th1-associated cytokines has been associated with corticosteroid-resistant interstitial lung disease in both PM and DM [21]. Therefore, in our previous study we sought to examine the IFN regulated chemokine and cytokine profiles in the serum of rituximab-treated refractory myositis patients, and explore the impact of rituximab on these cytokine/chemokine profiles at 8 and 16 weeks after rituximab treatment [12]. We find that the scores of Th2- and Th17-derived cytokines significantly increase at 16 weeks after rituximab, while the scores of regulatory cytokines decrease when compared with baseline level [12]. Interestingly, the IFN chemokine score does not differ significantly from baseline at either 8 or 16 weeks after initiation of rituximab [12]. In this study we demonstrate that patients with a high IFN chemokine score and high IL-2 levels at baseline experience a clinical improvement at 24 weeks post-rituximab in terms of Physician Global VAS and Muscle Disease Activity VAS.

Earlier studies have analyzed myositis autoantibodies as potential predictors of clinical improvement after initiation of rituximab in refractory myositis. Presence of anti-synthetases (mainly anti-Jo-1) and anti-Mi-2 were the strongest predictors of clinical improvement in a cohort of rituximab-treated myositis patients [7]. In our current study, presence or absence of autoantibodies was not predictive of Physician Global VAS or Muscle Disease Activity VAS at 24 weeks. However, in both "final” models of Physician Global VAS and Muscle Disease Activity VAS, the IFN chemokine score and IL-2 levels were predictors to some extent. Since the predic tive ability of the models including gene expression/cytokine/chemokine data was superior to that of the models without gene expression/cytokine/chemokine data, our findings suggest that gene expression/cytokine/hemone 
data, such as the IFN chemokine score and IL-2 levels, have greater predictive ability than autoantibody status for clinical improvement in terms of Physician Global and Muscle Disease Activity VAS. Nevertheless, our study also showed that presence of anti-Mi-2 and MIP- $1 \beta$ levels at baseline predict somewhat Extramuscular Global VAS at 24 weeks. These findings suggest that autoantibody status in conjunction with biomarkers such as MIP- $1 \beta$ could be beneficial for assessing clinical improvement in terms of Extramuscular Global VAS after rituximab therapy in patients with myositis.

Treatment strategies for DM and PM commonly include trials of medication based on provider preference and experience with disease features leading to multiple medication exposures and expense. Therapies such as rituximab have brought improved efficacy in the realm of autoimmune diseases, but their use in clinical practice may be limited by concerns over side effects, ineffectiveness and cost. Predictive models are needed to help identify patients with autoimmune diseases that would most likely benefit from rituximab therapy. Therefore, in this study, we sought to determine clinical, laboratory, and gene expression/cytokine/chemokine predictors of clinical improvement in rituximab-treated refractory myositis patients, and explore whether inclusion of gene expression/cytokine/chemokine data into the regression models offers a predictive advantage compared to models with clinical and laboratory data only. Interestingly, we found that "final" models that included gene expression/cytokine/chemokine data were superior in terms of predictive ability when compared to the "base" models with only clinical and laboratory data. Furthermore, our study indicates that IFN chemokine score and IL-2 levels predict to some extent clinical improvement in terms of both Physician Global VAS and Muscle Disease Activity VAS in rituximab-treated refractory myositis patients. Nevertheless, the penalized regression methods resulted in multivariable models with relatively low cross-validated $R^{2}$ values. This indicates that any predictive model built from the baseline variables available in our study is unlikely to demonstrate good performance when applied to new patients. Therefore, future studies should explore further the utility of gene expression, cytokine and chemokine data for predicting improvement in refractory myositis patients after initiating therapy with rituximab.

\section{Conflict of Interest}

The authors declare no conflicts of interest.

\section{Acknowledgements}

We would like to thank Peter Wettstein, $\mathrm{PhD}$ and Michael Strausbauch from the Immune Monitoring Laboratory, Mayo Clinic, Rochester, MN, for their roles in generating the cytokine/chemokine data. Also, we would like to thank Diane Koontz, University of Pittsburgh Medical Center, Pittsburgh, PA for the coordination of sample retrieval and shipment. Lastly, we would like to thank the RIM investigators and subjects. This work was also supported by Genentech.

\section{Funding Statement}

This work was supported by the National Institute of Health: Mechanisms of Response and Relapse in Rituximab-treated Refractory Idiopathic Inflammatory Myopathies [AR61298-03]; the National Institute of Arthritis and Musculoskeletal and Skin Diseases [N01-AR-4-2273, R01 AR061298, R01 AR57781], and the National Center for Advancing Translational Sciences [UL1 TR000135]. This work was also supported by The Myositis Association (TMA) and Genentech.

\section{References}

[1] Mastaglia, F.L., Garlepp, M.J., Phillips, B.A. and Zilko, P.J. (2003) Inflammatory Myopathies: Clinical, Diagnostic and Therapeutic Aspects. Muscle Nerve, 27, 407-425.

[2] Hoogendijk, J.E., Amato, A.A., Lecky, B.R., Choy, E.H., Lundberg, I.E., Rose, M.R., et al. (2004) Trial Design in Adult Idiopathic Inflammatory Myopathies, with the Exception of Inclusion Body Myositis. 119th ENMC International Workshop, Naarden, 10-12 October 2003, 337-345.

[3] Rider, L.G. and Miller, F.W. (1997) Classification and Treatment of the Juvenile Idiopathic Inflammatory Myopathies. Rheumatic Disease Clinics of North America, 23, 619-655.

[4] Nalotto, L., Iaccarino, L., Zen, M., Gatto, M., Borella, E., Domenighetti, M., et al. (2013) Rituximab in Refractory 
Idiopathic Inflammatory Myopathies and Antisynthetase Syndrome: Personal Experience and Review of the Literature. Immunologic Research, 56, 362-370. http://dx.doi.org/10.1007/s12026-013-8408-9

[5] Oddis, C.V., Reed, A.M., Aggarwal, R., Rider, L.G., Ascherman, D.P., Levesque, M.C., et al. (2013) Rituximab in the Treatment of Refractory Adult and Juvenile Dermatomyositis and Adult Polymyositis: A Randomized, Placebo-Phase Trial. Arthritis and Rheumatism, 65, 314-324.

[6] Stone, J.H., Merkel, P.A., Spiera, R., Seo, P., Langford, C.A., Hoffman, G.S., et al. (2010) Rituximab versus Cyclophosphamide for ANCA-Associated Vasculitis. New England Journal of Medicine, 363, 221-232.

[7] Aggarwal, R., Reed, A.M., Ascherman, D., Barohn, R., Feldman, B., et al. (2013) Clinical and Serologic Predictors of Response in Rituximab-Treated Refractory Adult and Juvenile Dermatomyositis and Adult Polymyositis.

[8] Lundberg, I., Ulfgren, A.K., Nyberg, P., Andersson, U. and Klareskog, L. (1997) Cytokine Production in Muscle Tissue of Patients with Idiopathic Inflammatory Myopathies. Arthritis and Rheumatism, 40, 865-874.

[9] Aleksza, M., Szegedi, A., Antal-Szalmas, P., Irinyi, B., Gergely, L., Ponyi, A., et al. (2005) Altered Cytokine Expression of Peripheral Blood Lymphocytes in Polymyositis and Dermatomyositis. Annals of the Rheumatic Diseases, 64, 1485-1489.

[10] Bilgic, H., Ytterberg, S.R., Amin, S., McNallan, K.T., Wilson, J.C., Koeuth, T., et al. (2009) Interleukin-6 and Type I Interferon-Regulated Genes and Chemokines Mark Disease Activity in Dermatomyositis. Arthritis and Rheumatism, 60, 3436-3446.

[11] Szodoray, P., Alex, P., Knowlton, N., Centola, M., Dozmorov, I., Csipo, I., et al. (2010) Idiopathic Inflammatory Myopathies, Signified by Distinctive Peripheral Cytokines, Chemokines and the TNF Family Members B-Cell Activating Factor and a Proliferation Inducing Ligand. Rheumatology, 49, 1867-1877. http://dx.doi.org/10.1093/rheumatology/keq151

[12] de Padilla, C.L., Crowson, C.S., Hein, M.S., Khun, H., Aggarwal, R., Levesque, M.C., et al. (2013) Interferon-Regulated Chemokine and Innate Cytokine Scores Identify Refractory Myositis Patients That Respond Better to Rtuximab Therapy. Arthritis and Rheumatism, 65, S887-S888.

[13] Reed, A.M., Peterson, E., Bilgic, H., Ytterberg, S.R., Amin, S., Hein, M.S., et al. (2012) Changes in Novel Biomarkers of Disease Activity in Juvenile and Adult Dermatomyositis Are Sensitive Biomarkers of Disease Course. Arthritis and Rheumatism, 64, 4078-4086.

[14] Bauer, J.W., Batliwalla, F.M., Wilson, J.C., Koeuth, T., Singh, S., et al. (2008) Baseline IFN-Regulated Chemokine Levels Are Strong Predictors of Systemic Lupus Erythematosus Flare (Abstract). Arthritis Rheumatology, 58, S805.

[15] Lundberg, I., Ulfgren, A.K., Nyberg, P., Andersson, U. and Klareskog, L. (1997) Cytokine Production in Muscle Tissue of Patients with Idiopathic Inflammatory Myopathies. Arthritis Rheumatology, 40, 865-874.

[16] Chinoy, H., Salway, F., John, S., Fertig, N., Tait, B.D., Oddis, C.V., et al. (2007) Interferon-Gamma and Interleukin-4 Gene Polymorphisms in Caucasian Idiopathic Inflammatory Myopathy Patients in UK. Annals of the Rheumatic Diseases, 66, 970-973.

[17] Tournadre, A., Lenief, V. and Miossec, P. (2010) Expression of Toll-Like Receptor 3 and Toll-Like Receptor 7 in Muscle Is Characteristic of Inflammatory Myopathy and Is Differentially Regulated by Th1 and Th17 Cytokines. Arthritis and Rheumatism, 62, 2144-2151.

[18] Shen, H., Xia, L., Lu, J. and Xiao, W. (2011) Interleukin-17 and Interleukin-23 in Patients with Polymyositis and Dermatomyositis. Scandinavian Journal of Rheumatology, 40, 217-220. http://dx.doi.org/10.3109/03009742.2010.517215

[19] Tibshirani, R. (1996) Regression Shrinkage and Selection via the Lasso. Journal of the Royal Statistical Society, 58, 267-288.

[20] Lundberg, I.E., Barbasso, S., Ulfgren, A.K., Gracie, J.A. and McInnes, I.B. (2005) Expression of IL-18 in Muscle Tissue of Patients with Treatment-Resistant Idiopathic Inflammatory Myopathies. Arthritis Research Therapy, 7, 57.

[21] Kurasawa, K., Nawata, Y., Takabayashi, K., Kumano, K., Kita, Y., Takiguchi, Y., et al. (2002) Activation of Pulmonary T Cells in Corticosteroid-Resistant and -Sensitive Interstitial Pneumonitis in Dermatomyositis/Polymyositis. Clinical Experimental Immunology, 129, 541-548. 


\section{Appendix}

\section{Predicting Improvement Using “Final” Multivariable Linear Regression Models (with Gene Expression/Cytokine/Chemokine Data)}

The following examples illustrate the direct application of our multivariable linear regression models in the clinical setting such as when a clinician is considering rituximab for a refractory myositis patient.

General formula for predicting absolute change in Physician Global VAS 24 weeks after rituximab treatment:

$$
y=1.36-0.34 x_{1}-0.26 x_{2}+1.10 x_{3}
$$

where $y$ is the estimated absolute change in Physician Global VAS 24 weeks after rituximab treatment, $x_{1}$ is the estimated regression coefficient of baseline Physician Global VAS, $x_{2}$ is the estimated regression coefficient of baseline IFN chemokine score, and $x_{3}$ is the estimated regression coefficient of baseline IL-2.

Case 1-A 40-year-old woman with newly diagnosed dermatomyositis presents with baseline Physician Global VAS of $40 \mathrm{~mm}$, IFN chemokine score of 55.7, and IL-2 of $3.5 \mathrm{pg} / \mathrm{mL}$. The estimated absolute change in Physician Global VAS 24 weeks after rituximab based on our multivariable linear regression model is computed as follows:

$$
y=1.36-0.34(40)-0.26(55.7)+1.10(3.5)=-22.9
$$

In this example, the estimated absolute change in Physician Global VAS 24 weeks after rituximab treatment is $-22.9 \mathrm{~mm}$. So by 24 weeks this patient's Physician Global VAS is estimated to decrease to $40.0-22.9=17.1$ $\mathrm{mm}$. Since her baseline Physician Global VAS was $40 \mathrm{~mm}$, we can say that she could experience around a 50\% reduction in her Physician Global VAS by 24 weeks if she were treated with rituximab. Therefore, this hypothetical patient could be a potential candidate for rituximab.

Case 2-A 61-year-old woman with newly diagnosed dermatomyositis presents with baseline Physician Global VAS of $12 \mathrm{~mm}$, IFN chemokine score of 1.9, and IL-2 of $2.2 \mathrm{pg} / \mathrm{mL}$. The estimated absolute change in Physician Global VAS 24 weeks after rituximab based on our multivariable linear regression model is computed as follows:

$$
y=1.36-0.34(12)-0.26(1.9)+1.10(2.2)=-0.8
$$

In this example, the estimated absolute change in Physician Global VAS 24 weeks after rituximab treatment is $-0.8 \mathrm{~mm}$. So by 24 weeks this patient's Physician Global VAS is estimated to decrease to $12.0-0.8=11.2$, which is very little change from baseline. Since the benefit from rituximab is not significant in this case, this hypothetical patient would not be an ideal candidate for rituximab. 